\title{
The homotopy types of SO(4)-gauge groups
}

\section{Daisuke Kishimoto $^{1} \cdot$ Ingrid Membrillo-Solis $^{2} \cdot$ Stephen Theriault $^{2}$}

Received: 6 June 2020 / Revised: 9 November 2020 / Accepted: 25 November 2020 /

Published online: 27 April 2021

(C) The Author(s) 2021

\begin{abstract}
The homotopy types of gauge groups of principal SO(4)-bundles over $S^{4}$ are classified $p$-locally for every prime $p$, and partial results are obtained integrally. The method generalizes to deal with any quotient of the form $\left(S^{3}\right)^{n} / Z$ where $Z$ is a subgroup generated by $(-1, \ldots,-1)$.
\end{abstract}

Keywords $\mathrm{SO}(4) \cdot$ Gauge group $\cdot$ Homotopy type

Mathematics Subject Classification 55P15 · 54C35 · 81T13

\section{Introduction}

Let $G$ be a topological group and $P$ be a principal $G$-bundle over a base space $X$. The gauge group of $P$ is the topological group of $G$-equivariant automorphisms of $P$ which cover the identity map on $X$. If $X$ is a finite $C W$-complex, Crabb and Sutherland [2] showed that, despite there possibly being infinitely many inequivalent principal $G$ bundles over $X$, there are only finitely many homotopy types for the corresponding gauge groups. There has been an intensive effort recently to classify the homotopy types of gauge groups, particularly in cases of interest to physics and geometry.

The first author is supported by JSPS KAKENHI (No. 17K05248), the second author is supported by EPSRC Grant EP/N014189/1 and Leverhulme Trust Grant RPG-2019-055.

\footnotetext{
$凶 \quad$ Stephen Theriault

S.D.Theriault@soton.ac.uk

Daisuke Kishimoto

kishi@math.kyoto-u.ac.jp

Ingrid Membrillo-Solis

I.Membrillo-Solis@ soton.ac.uk

1 Department of Mathematics, Kyoto University, Kyoto 606-8502, Japan

2 Mathematical Sciences, University of Southampton, Southampton SO17 1BJ, UK
} 
In this paper, we consider the homotopy types of gauge groups of principal SO(4)bundles over $S^{4}$. Our results are stated in more generality. Let $Z$ be a subgroup of $\left(S^{3}\right)^{n}$ generated by an element $(-1, \ldots,-1)$. Define

$$
K_{n}=\left(S^{3}\right)^{n} / Z
$$

Then, in particular, $K_{1}=\mathrm{SO}(3)$ and $K_{2}=\mathrm{SO}(4)$. Let $\epsilon_{i}$ be the composite of the $i$-th inclusion $S^{3} \rightarrow\left(S^{3}\right)^{n}$ and the projection $\left(S^{3}\right)^{n} \rightarrow K_{n}$ for $1 \leqslant i \leqslant n$. Then $\pi_{3}\left(K_{n}\right) \cong \mathbb{Z}^{n}$ is generated by $\epsilon_{1}, \ldots, \epsilon_{n}$. Let $\mathcal{G}_{k_{1}, \ldots, k_{n}}$ be the gauge group of a principal $K_{n}$-bundle over $S^{4}$ corresponding to $k_{1} \epsilon_{1}+\cdots+k_{n} \epsilon_{n} \in \pi_{3}\left(K_{n}\right)$. The aim of this paper is to classify the homotopy types of gauge groups $\mathcal{G}_{k_{1}, \ldots, k_{n}}$ as $k_{1}, \ldots, k_{n}$ range over all integers.

Let $\left\{a_{1}, \ldots, a_{n}\right\}$ denote a multiset consisting of elements $a_{1}, \ldots, a_{n}$. For integers $a$ and $b$, let $(a, b)$ be their greatest common divisor.

Theorem 1.1 The following hold:

(a) if $\left\{\left(k_{1}, 12\right), \ldots,\left(k_{n}, 12\right)\right\}=\left\{\left(l_{1}, 12\right), \ldots,\left(l_{n}, 12\right)\right\}$ then there is a homotopy equivalence $\mathcal{G}_{k_{1}, \ldots, k_{n}} \simeq \mathcal{G}_{l_{1}, \ldots, l_{n}}$;

(b) if there is a homotopy equivalence $\mathcal{S}_{k_{1}, \ldots, k_{n}} \simeq \mathcal{G}_{l_{1}, \ldots, l_{n}}$ then $\left\{\left(k_{1}, 4\right), \ldots,\left(k_{n}, 4\right)\right\}=$ $\left\{\left(l_{1}, 4\right), \ldots,\left(l_{n}, 4\right)\right\}$ and $\left\{\left(k_{1}, 3\right), \ldots,\left(k_{n}, 3\right)\right\}=\left\{\left(l_{1}, 3\right), \ldots,\left(l_{n}, 3\right)\right\}$.

The weaker statement in Theorem 1.1(b) stems from the fact that the homotopy equivalence induces an isomorphism of homotopy groups, but as we will see in Lemma 2.7 , the isomorphism of second homotopy groups may take the form $\mathbb{Z} / 12 \oplus \mathbb{Z} / 1 \cong$ $\mathbb{Z} / 3 \oplus \mathbb{Z} / 4$ when $n=2$ (where $\mathbb{Z} / 1$ is the trivial group), which does not imply an equality between $\{(1,12),(12,12)\}$ and $\{(4,12),(3,12)\}$.

If one is willing to localize then a classification holds. For a nilpotent space $X$ and a prime $p$, let $X_{(p)}$ be the localization of $X$ at $p$. For an integer $m$, let $v_{p}(m)$ be the $p$-component of $m$.

Theorem 1.2 Let $p$ be a prime. There is a p-local homotopy equivalence

$$
\left(\mathcal{G}_{k_{1}, \ldots, k_{n}}\right)_{(p)} \simeq\left(\mathcal{G}_{l_{1}, \ldots, l_{n}}\right)_{(p)}
$$

if and only if $\left\{v_{p}\left(\left(k_{1}, 12\right)\right), \ldots, v_{p}\left(\left(k_{n}, 12\right)\right)\right\}=\left\{v_{p}\left(\left(l_{1}, 12\right)\right), \ldots, v_{p}\left(\left(l_{n}, 12\right)\right)\right\}$.

The $K_{1}=\mathrm{SO}(3)$ case in Theorem 1.1 is already known [7]. In this case, as there is only one index involved, Theorem 1.1 implies the stronger statement that $\mathcal{G}_{k} \simeq \mathcal{G}_{l}$ if and only if $(k, 12)=(l, 12)$. The key new case is for $K_{2}=\mathrm{SO}(4)$.

The $\mathrm{SO}(4)$ and Spin (4) cases are the last to consider among the principal $G$-bundles over $S^{4}$ when $G$ is a connected, compact Lie group of type 2 . A classification in the $\mathrm{SU}(3)$ case was completed in [6] and the PU(3) case in [5], a classification of the $p$-local homotopy types in the $\operatorname{Sp}(2)$ case was completed in [10] and $\operatorname{PSp}(2)$ in [5], the $U(2)$-case was classified in [3], and the $p$-local homotopy types in the $G_{2}$ case were classified up to one factor of 2 in [8].

The overall strategy used to prove Theorem 1.1 is similar to that in the other type 2 cases, but distinctive features arise. The principal SO(4)-bundles over $S^{4}$ are in oneto-one correspondence with $\left[S^{4}, B S O(4)\right] \cong \mathbb{Z} \oplus \mathbb{Z}$ and so require a multi-index, as 
opposed to the earlier cases which only required a single index. This leads to the use of multisets and the possibility that a homotopy equivalence $\mathcal{G}_{k_{1}, k_{2}} \simeq \mathcal{G}_{l_{1}, l_{2}}$ may arise via a permutation of indices. It also leads to the issue mentioned above of a group decomposition preventing an identification of multisets.

\section{Proof of Theorem 1.1}

Recall from [4] (cf. [1]) that there is a homotopy equivalence

$$
B \mathcal{G}_{k_{1}, \ldots, k_{n}} \simeq \operatorname{map}\left(S^{4}, B K_{n} ; k_{1} \epsilon_{1}+\cdots+k_{n} \epsilon_{n}\right)
$$

where the right-hand side is the path-connected component of the space of maps from $S^{4}$ to $B K_{n}$ containing $k_{1} \epsilon_{1}+\cdots+k_{n} \epsilon_{n}$. Consider the homotopy fiber sequence

$$
K_{n} \stackrel{\partial_{k_{1}, \ldots, k_{n}}}{\longrightarrow} \Omega_{0}^{3} K_{n} \rightarrow \operatorname{map}\left(S^{4}, B K_{n} ; k_{1} \epsilon_{1}+\cdots+k_{n} \epsilon_{n}\right) \rightarrow B K_{n},
$$

where the last map is the evaluation at the basepoint. Then $\mathcal{G}_{k_{1}, \ldots, k_{n}}$ is homotopy equivalent to the homotopy fiber of $\partial_{k_{1}, \ldots, k_{n}}$. Let $\pi: K_{n} \rightarrow K_{1}^{n}$ be the canonical projection. Since $\Omega^{3} \pi: \Omega_{0}^{3} K_{n} \rightarrow\left(\Omega_{0}^{3} K_{1}\right)^{n}$ is a homotopy equivalence, one obtains the following.

Lemma 2.1 The gauge group $\mathcal{G}_{k_{1}, \ldots, k_{n}}$ is homotopy equivalent to the homotopy fiber of $\Omega^{3} \pi \circ \partial_{k_{1}, \ldots, k_{n}}$.

We need to identify the map $\Omega^{3} \pi \circ \partial_{k_{1}, \ldots, k_{n}}$. Whitehead [11] showed that the adjoint $S^{4} \wedge K_{n} \rightarrow B K_{n}$ of $\partial_{k_{1}, \ldots, k_{n}}$ is the Whitehead product of the adjoint of $k_{1} \epsilon_{1}+\cdots+k_{n} \epsilon_{n}$ and the canonical inclusion $\Sigma K_{n} \rightarrow B K_{n}$. Thus by the adjointness of Whitehead products and Samelson products, we obtain the following.

Lemma 2.2 The adjoint $S^{3} \wedge K_{n} \rightarrow K_{n}$ of the map $\partial_{k_{1}, \ldots, k_{n}}$ is the Samelson product $\left\langle k_{1} \epsilon_{1}+\cdots+k_{n} \epsilon_{n}, 1_{K_{n}}\right\rangle$.

Now we calculate $\pi \circ\left\langle k_{1} \epsilon_{1}+\cdots+k_{n} \epsilon_{n}, 1_{K_{n}}\right\rangle$. For $1 \leqslant i \leqslant n$, let $p_{i}: K_{1}^{n} \rightarrow K_{1}$ be the projection onto the $i^{\text {th }}$-factor. Define $\pi_{i}$ by the composite

$$
\pi_{i}: K_{n} \stackrel{\pi}{\longrightarrow} K_{1}^{n} \stackrel{p_{i}}{\longrightarrow} K_{1}
$$

Define $\bar{\epsilon}_{i}$ and $\lambda_{i}$ by the composites

$$
\bar{\epsilon}_{i}: S^{3} \stackrel{\epsilon_{i}}{\longrightarrow} K_{n} \stackrel{\pi_{i}}{\longrightarrow} K_{1}, \quad \lambda_{i}: S^{3} \wedge K_{n} \stackrel{\left\langle\bar{\epsilon}_{i}, \pi_{i}\right\rangle}{\longrightarrow} K_{1}
$$

Observe that $\pi_{i} \circ\left(k_{1} \epsilon_{1}+\cdots+k_{n} \epsilon_{n}\right) \simeq k_{i} \bar{\epsilon}_{i}$. Therefore, as $\pi_{i}$ is a homomorphism, $\pi_{i} \circ\left\langle k_{1} \epsilon_{1}+\cdots+k_{n} \epsilon_{n}, 1_{K_{n}}\right\rangle \simeq\left\langle\pi_{i} \circ\left(k_{1} \epsilon_{1}+\cdots+k_{n} \epsilon_{n}\right), \pi_{i}\right\rangle \simeq k_{i}\left\langle\bar{\epsilon}_{i}, \pi_{i}\right\rangle=k_{i} \lambda_{i}$. 
Thus $\pi \circ\left\langle k_{1} \epsilon_{1}+\cdots+k_{n} \epsilon_{n}, 1_{K_{n}}\right\rangle \simeq\left(k_{1} \lambda_{1}, \ldots, k_{n} \lambda_{n}\right)$. Let $k: K_{1} \rightarrow K_{1}$ be the $k^{\text {th }}$-power map. Then $\left(k_{1} \lambda_{1}, \ldots, k_{n} \lambda_{n}\right) \simeq\left(k_{1} \times \cdots \times k_{n}\right) \circ\left(\lambda_{1}, \ldots, \lambda_{n}\right)$. Hence the composite $\pi \circ\left\langle k_{1} \epsilon_{1}+\cdots+k_{n} \epsilon_{n}, 1_{K_{n}}\right\rangle$ has the following linearity property.

Proposition 2.3 There is a homotopy

$$
\pi \circ\left\langle k_{1} \epsilon_{1}+\cdots+k_{n} \epsilon_{n}, 1_{K_{n}}\right\rangle \simeq\left(k_{1} \times \cdots \times k_{n}\right) \circ\left(\lambda_{1}, \ldots, \lambda_{n}\right) .
$$

Next, we determine the order of $\lambda_{i}$.

Proposition 2.4 The order of $\lambda_{i}=\left\langle\bar{\epsilon}_{i}, \pi_{i}\right\rangle$ is 12 for each $1 \leqslant i \leqslant n$.

Proof The diagonal map $S^{3} \rightarrow\left(S^{3}\right)^{n}$ induces an inclusion $j: K_{1} \rightarrow K_{n}$ which is a section of $\pi_{i}$ for each $1 \leqslant i \leqslant n$. On the one hand, since $\pi_{i} \circ j$ is the identity map on $K_{1}$, the composite

$$
S^{3} \wedge K_{1} \stackrel{1_{S^{3} \wedge j}}{\longrightarrow} S^{3} \wedge K_{n} \stackrel{\left\langle\bar{\epsilon}_{i}, \pi_{i}\right\rangle}{\longrightarrow} K_{1}
$$

is $\left\langle\bar{\epsilon}_{i}, 1_{K_{1}}\right\rangle$. By [7] the order of $\left\langle\bar{\epsilon}_{i}, 1_{K_{1}}\right\rangle$ is 12. Thus the order of $\left\langle\bar{\epsilon}_{i}, \pi_{i}\right\rangle$ is at least 12. On the other hand, observe that $\left\langle\bar{\epsilon}_{i}, \pi_{i}\right\rangle$ factors as the composite

$$
S^{3} \wedge K_{n} \stackrel{1_{S^{3}} \wedge \pi_{i}}{\longrightarrow} S^{3} \wedge K_{1} \stackrel{\left\langle\bar{\epsilon}_{i}, 1_{K_{1}}\right\rangle}{\longrightarrow} K_{1} .
$$

Thus the order of $\left\langle\bar{\epsilon}_{i}, \pi_{i}\right\rangle$ is at most 12 . Hence the order of $\left\langle\bar{\epsilon}_{i}, \pi_{i}\right\rangle$ is precisely 12 .

Lemma 2.5 Let $\alpha_{i}: X \rightarrow Y_{i}$ be a map of order $p^{m_{i}}$ into a $p$-local $H$-space $Y_{i}$ for $1 \leqslant i \leqslant n$. If $\left(k_{i}, p^{m_{i}}\right)=\left(l_{i}, p^{m_{i}}\right)$ for all $i$, then there is a self-homotopy equivalence $h_{p}$ of $Y_{1} \times \cdots \times Y_{n}$ such that

$$
h_{p} \circ\left(k_{1} \times \cdots \times k_{n}\right) \circ\left(\alpha_{1}, \ldots, \alpha_{n}\right) \simeq\left(l_{1} \times \cdots \times l_{n}\right) \circ\left(\alpha_{1}, \ldots, \alpha_{n}\right) .
$$

Proof If $\left(k_{i}, p^{m_{i}}\right)=\left(l_{i}, p^{m_{i}}\right)=p^{m_{i}}$ then the composites $k_{i} \circ \alpha_{i}$ and $l_{i} \circ \alpha_{i}$ are both null homotopic since $\alpha_{i}$ has order $p^{m_{i}}$. In that case let $h_{p, i}$ be the identity map on $Y_{i}$. If $\left(k_{i}, p^{m_{i}}\right)=\left(l_{i}, p^{m_{i}}\right)<p^{m_{i}}$, let $a_{i}=\frac{k_{i}}{\left(k_{i}, p^{m_{i}}\right)}$ and $b_{i}=\frac{l_{i}}{\left(l_{i}, p^{m_{i}}\right)}$. Then $a_{i}$ and $b_{i}$ are units in $\mathbb{Z}_{(p)}$, and in particular, the power maps $a_{i}, b_{i}: Y_{i} \rightarrow Y_{i}$ are homotopy equivalences. As $\left(k_{i}, p^{m_{i}}\right)=\left(l_{i}, p^{m_{i}}\right)$ we obtain $\frac{b_{i}}{a_{i}} k_{i}=l_{i}$. Therefore if $h_{p, i}=$ $b_{i} \circ a_{i}^{-1}$ then $h_{p, i} \circ k_{i} \simeq l_{i}$ as self-maps of $Y_{i}$. Thus $h_{p}=h_{p, 1} \times \cdots \times h_{p, n}$ is the desired self-homotopy equivalence.

Proposition 2.6 If $\left\{\left(k_{1}, 12\right), \ldots,\left(k_{n}, 12\right)\right\}=\left\{\left(l_{1}, 12\right), \ldots,\left(l_{n}, 12\right)\right\}$, then $\mathcal{G}_{k_{1}, \ldots, k_{n}}$ and $\mathcal{G}_{l_{1}, \ldots, l_{n}}$ are homotopy equivalent.

Proof By assumption, there is a permutation $\sigma$ such that $\left(\left(k_{\sigma(1)}, 12\right), \ldots,\left(k_{\sigma(n)}, 12\right)\right)=$ $\left(\left(l_{1}, 12\right), \ldots,\left(l_{n}, 12\right)\right)$. We denote the permutation of $\left(S^{3}\right)^{n}$ induced from $\sigma$ by the same symbol. This automorphism induces an automorphism of $K_{n}$ which we denote by $\bar{\sigma}$. The automorphism $\bar{\sigma}$ induces a homotopy commutative diagram of Samelson products 


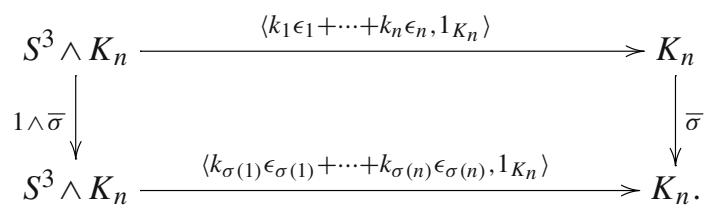

Taking adjoints, by Lemma 2.2 we obtain $\Omega^{3} \bar{\sigma} \circ \partial_{k_{1}, \ldots, k_{n}} \simeq \partial_{k_{\sigma(1)}, \ldots, k_{\sigma(n)}} \circ \bar{\sigma}$. Composing with the map $\Omega^{3} K \stackrel{\Omega^{3} \pi}{\longrightarrow} \Omega^{3} K_{1}^{n}$ and using the fact that $\Omega_{0}^{3} K_{1}^{n} \simeq\left(\Omega_{0}^{3} S^{3}\right)^{n}$, we obtain a homotopy commutative diagram

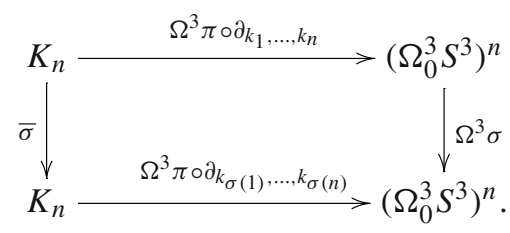

By Lemma 2.1, the homotopy fibre of $\Omega^{3} \pi \circ \partial_{k_{1}, \ldots, k_{n}}$ is $\mathcal{G}_{k_{1}, \ldots, k_{n}}$. So as $\sigma$ and $\bar{\sigma}$ are homotopy equivalences, this diagram induces a homotopy equivalence $\mathcal{G}_{k_{1}, \ldots, k_{n}} \simeq$ $\mathcal{G}_{k_{\sigma(1)}, \ldots, k_{\sigma(n)}}$. Thus, for ease of notation in what follows, we may assume without loss of generality that the permutation $\sigma$ is the identity.

By Proposition 2.3, $\pi \circ\left\langle k_{1} \epsilon_{1}+\cdots+k_{n} \epsilon_{n}, 1_{K_{n}}\right\rangle \simeq\left(k_{1} \times \cdots \times k_{n}\right) \circ\left(\lambda_{1}, \ldots, \lambda_{n}\right)$. Since $\pi_{i}\left(\Omega_{0}^{3} S^{3}\right)$ is finite for each $i$, there is a homotopy equivalence

$$
\Omega_{0}^{3} S^{3} \simeq \prod_{p \in P} \Omega_{0}^{3} S_{(p)}^{3}
$$

where $P$ is the set of all primes and $S_{(p)}^{3}$ is the localization of $S^{3}$ at $p$. Let $\widehat{\lambda}_{i}: K_{n} \rightarrow$ $\Omega_{0}^{3} S^{3}$ be the adjoint of $\lambda_{i}$ and let

$$
\left(\widehat{\lambda}_{1}, \ldots, \widehat{\lambda}_{n}\right)_{p}: K_{n} \rightarrow\left(\Omega_{0}^{3} S_{(p)}^{3}\right)^{n}
$$

be the composite of $\left(\widehat{\lambda}_{1}, \ldots, \widehat{\lambda}_{n}\right)$ and the map localizing $\left(\Omega_{0}^{3} S^{3}\right)^{n}$ to $\left(\Omega_{0}^{3} S_{(p)}^{3}\right)^{n}$. For convenience, the $m^{\text {th }}$-power map on $\Omega_{0}^{3} S^{3}$ will also be denoted by $m$. By Lemma 2.5 , for each prime $p$ there is a self-homotopy equivalence $h_{p}$ of $\left(\Omega_{0}^{3} S_{p}^{3}\right)^{n}$ satisfying a homotopy commutative diagram

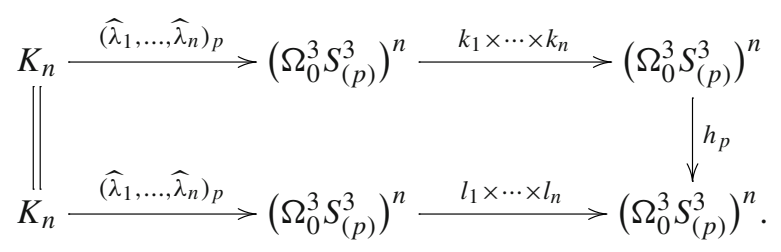


Note that since each $k_{i} \circ \widehat{\lambda}_{i}$ is a divisor of 12 , the map $h_{p}$ is a homotopy equivalence if $p \geqslant 5$. Let $h$ be the composite

$$
h:\left(\Omega_{0}^{3} S^{3}\right)^{n} \stackrel{\simeq}{\longrightarrow} \prod_{p \in P}\left(\Omega_{0}^{3} S_{(p)}^{3}\right)^{n} \stackrel{\prod_{p \in P} h_{p}}{\longrightarrow} \prod_{p \in P}\left(\Omega_{0}^{3} S_{(p)}^{3}\right)^{n} \stackrel{\simeq}{\longrightarrow}\left(\Omega_{0}^{3} S^{3}\right)^{n} .
$$

Since each $h_{p}$ is a homotopy equivalence, so is $h$. From diagram (2.1) at each $p$ we obtain a homotopy commutative diagram

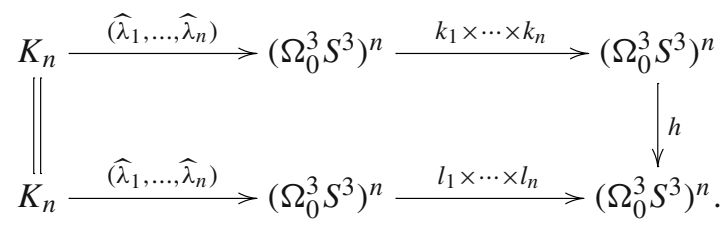

On the one hand, by definition, $\widehat{\lambda}_{i}$ is the adjoint of $\lambda_{i}$, so the adjoint of $\left(k_{1} \times \cdots \times k_{n}\right) \circ\left(\widehat{\lambda}_{1}, \ldots, \widehat{\lambda}_{n}\right)$ is $\left(k_{1} \times \cdots \times k_{n}\right) \circ\left(\lambda_{1}, \ldots, \lambda_{n}\right)$, which by Proposition 2.3 is homotopic to $\pi \circ\left\langle k_{1} \epsilon_{1}+\cdots+k_{n} \epsilon_{n}, 1_{K_{n}}\right\rangle$. On the other hand, by Lemma 2.2, $\pi \circ \partial_{k_{1}, \ldots, k_{n}}$ is also the adjoint of $\pi \circ\left\langle k_{1} \epsilon_{1}+\cdots+k_{n} \epsilon_{n}, 1_{K_{n}}\right\rangle$. This implies that $\left(k_{1} \times \cdots \times k_{n}\right) \circ\left(\widehat{\lambda}_{1}, \ldots, \widehat{\lambda}_{n}\right) \simeq \pi \circ \partial_{k_{1}, \ldots, k_{n}}$. Hence (2.2) may be rewritten as a homotopy commutative diagram

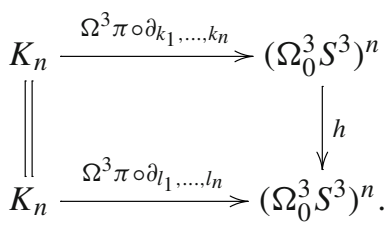

By Lemma 2.1, the homotopy fibres of $\Omega^{3} \pi \circ \partial_{k_{1}, \ldots, k_{n}}$ and $\Omega^{3} \pi \circ \partial_{k_{1}, \ldots, k_{n}}$ respectively are $\mathcal{S}_{k_{1}, \ldots, k_{n}}$ and $\mathcal{G}_{l_{1}, \ldots, l_{n}}$. From (2.3) there is an induced map of homotopy fibres $\mathcal{G}_{k_{1}, \ldots, k_{n}} \rightarrow \mathcal{G}_{l_{1}, \ldots, l_{n}}$. As $h$ is a homotopy equivalence, this induced map of homotopy fibres is also a homotopy equivalence, completing the proof.

Let $\tilde{\epsilon}_{i}: S^{3} \rightarrow\left(S^{3}\right)^{n}$ be the $i$-th inclusion for $1 \leqslant i \leqslant n$. Let $\widetilde{\mathscr{G}}_{k_{1}, \ldots, k_{n}}$ be the gauge group of a principal $\left(S^{3}\right)^{n}$-bundle over $S^{4}$ corresponding to $k_{1} \tilde{\epsilon}_{1}+\cdots+k_{n} \tilde{\epsilon}_{n}$. Then there is an isomorphism

$$
\widetilde{\mathcal{G}}_{k_{1}, \ldots, k_{n}} \cong \widetilde{\mathcal{G}}_{k_{1}} \times \cdots \times \widetilde{\mathcal{G}}_{k_{n}}
$$

where each $\widetilde{\mathcal{G}}_{k_{i}}$ is the gauge group of the principal $S^{3}$ bundle over $S^{4}$ classified by $k_{i} \in \mathbb{Z} \cong \pi_{4}\left(B S^{3}\right)$. 
On the other hand, there is a two-sheeted covering $\widetilde{\mathcal{G}}_{k_{1}, \ldots, k_{n}} \rightarrow \mathcal{G}_{k_{1}, \ldots, k_{n}}$. Therefore for all $m \geqslant 2$ there is an isomorphism

$$
\pi_{m}\left(\mathcal{G}_{k_{1}, \ldots, k_{n}}\right) \cong \bigoplus_{i=1}^{n} \pi_{m}\left(\widetilde{\mathcal{G}}_{k_{i}}\right)
$$

In particular, by [9], $\pi_{2}\left(\widetilde{\mathcal{G}}_{k}\right) \cong \mathbb{Z} / \frac{12}{(k, 12)}$, so we obtain the following.

Lemma $2.7 \pi_{2}\left(\mathcal{G}_{k_{1}, \ldots, k_{n}}\right) \cong \mathbb{Z} / \frac{12}{\left(k_{1}, 12\right)} \oplus \cdots \oplus \mathbb{Z} / \frac{12}{\left(k_{n}, 12\right)}$.

Observe that the isomorphism in Lemma 2.7 does not imply that there is an equality of multisets $\left\{\left(k_{1}, 12\right), \ldots,\left(k_{n}, 12\right)\right\}=\left\{\left(l_{1}, 12\right), \ldots,\left(l_{n}, 12\right)\right\}$. For example, if $n=2$ then $\mathbb{Z} / 12 \oplus \mathbb{Z} / 1 \cong \mathbb{Z} / 3 \oplus \mathbb{Z} / 4$ but $\{(1,12),(12,12)\} \neq\{(4,12),(3,12)\}$. However, if we work one prime at a time we do get an equality of multisets.

Proposition 2.8 If $\mathcal{G}_{k_{1}, \ldots, k_{n}} \simeq \mathcal{G}_{l_{1}, \ldots, l_{n}}$ then we obtain $\left\{\left(k_{1}, 4\right), \ldots,\left(k_{n}, 4\right)\right\}=$ $\left\{\left(l_{1}, 4\right), \ldots,\left(l_{n}, 4\right)\right\}$ and $\left\{\left(k_{1}, 3\right), \ldots,\left(k_{n}, 3\right)\right\}=\left\{\left(l_{1}, 3\right), \ldots,\left(l_{n}, 3\right)\right\}$.

Proof By Lemma 2.7, the homotopy equivalence $\mathcal{G}_{k_{1}, \ldots, k_{n}} \simeq \mathcal{G}_{l_{1}, \ldots, l_{n}}$ implies that there is an isomorphism of groups

$$
\mathbb{Z} / \frac{12}{\left(k_{1}, 12\right)} \oplus \cdots \oplus \mathbb{Z} / \frac{12}{\left(k_{n}, 12\right)} \cong \mathbb{Z} / \frac{12}{\left(l_{1}, 12\right)} \oplus \cdots \oplus \mathbb{Z} / \frac{12}{\left(l_{n}, 12\right)}
$$

For a prime $p$, let $\mathbb{Z}_{(p)}$ be the integers localized at $p$. Tensoring the isomorphism (2.5) with $\mathbb{Z}_{(2)}$ gives a group isomorphism

$$
\mathbb{Z} / \frac{4}{\left(k_{1}, 4\right)} \oplus \cdots \oplus \mathbb{Z} / \frac{4}{\left(k_{n}, 4\right)} \cong \mathbb{Z} / \frac{4}{\left(l_{1}, 4\right)} \oplus \cdots \oplus \mathbb{Z} / \frac{4}{\left(l_{n}, 4\right)}
$$

The groups $\mathbb{Z} / 1, \mathbb{Z} / 2$ and $\mathbb{Z} / 4$ appearing on either side of this isomorphism are indecomposable, so each side must have the same number of generators of each order. Hence $\left\{\left(k_{1}, 4\right), \ldots,\left(k_{n}, 4\right)\right\}=\left\{\left(l_{1}, 4\right), \ldots,\left(l_{n}, 4\right)\right\}$. The same argument applies if (2.5) is tensored with $\mathbb{Z}_{(3)}$.

Finally, we prove Theorems 1.1 and 1.2.

Proof of Theorem 1.1 Combine Propositions 2.6 and 2.8.

Proof of Theorem 1.2 Suppose there is a $p$-local homotopy equivalence $\left(\mathcal{G}_{k_{1}, \ldots, k_{n}}\right)_{(p)} \simeq$ $\left(\mathcal{G}_{l_{1}, \ldots, l_{n}}\right)_{(p)}$. Notice that $\nu_{2}\left(\left(k_{i}, 12\right)\right)=\left(k_{i}, 4\right)$ and $\nu_{3}\left(\left(k_{i}, 12\right)\right)=\left(k_{i}, 3\right)$, so Proposition 2.8 proves the $p=2$ and $p=3$ cases. If $p \geqslant 5$ then $v_{p}\left(\left(k_{i}, 12\right)\right)=1$ and $v_{p}\left(\left(l_{i}, 12\right)\right)=1$ for all $1 \leqslant i \leqslant n$, so the asserted equality of multisets holds. The converse is proved using the same argument as for Proposition 2.6; in fact, it is easier since we need only consider the factor $\Omega_{0}^{3} S_{(p)}^{3}$ of $\Omega_{0}^{3} S^{3}=\prod_{p \in P} \Omega_{0}^{3} S_{(p)}^{3}$. 
Remark 2.9 Some generalization is possible. Let $G$ be a simply-connected, simple compact Lie group with centre $Z(G)$. Let $L_{n}=G^{n} / Z$ where $Z$ is the subgroup generated by the image of the diagonal map $Z(G) \rightarrow Z(G)^{n}$. Replacing $K_{n}$ with $L_{n}$, the material in Sect. 2 through to Proposition 2.4 generalizes, where the order of $\lambda_{i}=\left\langle\bar{\epsilon}_{i}, \pi_{i}\right\rangle$ may no longer be 12 but it is a fixed number $M$ for all $1 \leqslant k \leqslant n$. The proof of Proposition 2.6 leading to (2.1) holds, giving the statement that if $\left\{\left(k_{1}, M\right), \ldots,\left(k_{n}, M\right)\right\}=\left\{\left(l_{1}, M\right), \ldots,\left(l_{n}, M\right)\right\}$ then $\mathcal{G}_{k_{1}, \ldots, k_{n}}$ and $\mathcal{G}_{l_{1}, \ldots, l_{n}}$ are $p$ locally homotopy equivalent for each prime $p$. However, as $\Omega_{0}^{3} G$ may not only have torsion homotopy groups, the argument for (2.2) leading to an integral homotopy equivalence of gauge groups will not hold. Further, the converse statement in Proposition 2.8 requires the homotopy type of $\widetilde{\mathcal{G}}_{k}$ to be determined by a homotopy set that depends on $(k, M)$, which is not known to hold in general.

Acknowledgements The authors would like to thank the referee for a careful reading of the paper that has improved its exposition.

Open Access This article is licensed under a Creative Commons Attribution 4.0 International License, which permits use, sharing, adaptation, distribution and reproduction in any medium or format, as long as you give appropriate credit to the original author(s) and the source, provide a link to the Creative Commons licence, and indicate if changes were made. The images or other third party material in this article are included in the article's Creative Commons licence, unless indicated otherwise in a credit line to the material. If material is not included in the article's Creative Commons licence and your intended use is not permitted by statutory regulation or exceeds the permitted use, you will need to obtain permission directly from the copyright holder. To view a copy of this licence, visit http://creativecommons.org/licenses/by/4.0/.

\section{References}

1. Atiyah, M.F., Bott, R.: The Yang-Mills equations over Riemann surfaces. Philos. Trans. Roy. Soc. London Ser. A 308(1505), 523-615 (1983)

2. Crabb, M.C., Sutherland, W.A.: Counting homotopy types of gauge groups. Proc. London Math. Soc. 81(3), 747-768 (2000)

3. Cutler, T.: The homotopy types of $U(n)$-gauge groups over $S^{4}$ and $\mathbb{C} P^{2}$. Homology, Homot. Appl. 20(1), 5-36 (2018)

4. Gottlieb, D.H.: Applications of bundle map theory. Trans. Amer. Math. Soc. 171, 23-50 (1972)

5. Hasui, S., Kishimoto, D., Kono, A., Sato, T.: The homotopy types of PU(3) and PSp(2)-gauge groups. Algebr. Geom. Topol. 16(3), 1813-1825 (2016)

6. Hamanaka, H., Kono, A.: Unstable $K^{1}$-group and homotopy type of certain gauge groups. Proc. Roy. Soc. Edinburgh Sect. A 136(1), 149-155 (2006)

7. Kamiyama, Y., Kishimoto, D., Kono, A., Tsukuda, S.: Samelson products of SO(3) and applications. Glasgow Math. J. 49(2), 405-409 (2007)

8. Kishimoto, D., Theriault, S., Tsutaya, M.: The homotopy types of $G_{2}$-gauge groups. Topol. Appl. 228, 92-107 (2017)

9. Kono, A.: A note on the homotopy type of certain gauge groups. Proc. Roy. Soc. Edinburgh Sect. A 117(3), 295-297 (1991)

10. Theriault, S.D.: The homotopy types of Sp(2)-gauge groups. Kyoto J. Math. 50(3), 591-605 (2010)

11. Whitehead, G.W.: On products in homotopy groups. Ann. Math. 47(3), 460-475 (1946)

Publisher's Note Springer Nature remains neutral with regard to jurisdictional claims in published maps and institutional affiliations. 